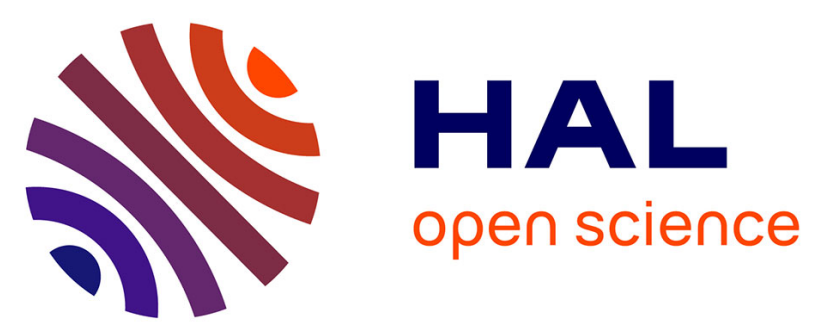

\title{
Static electric field enhancement in nanoscale structures
}

Bruno Lepetit, Didier Lemoine, Maykel Márquez-Mijares

\section{To cite this version:}

Bruno Lepetit, Didier Lemoine, Maykel Márquez-Mijares. Static electric field enhancement in nanoscale structures. Journal of Applied Physics, 2016, 120, pp.085105. 10.1063/1.4961216 . hal01361782

\section{HAL Id: hal-01361782 \\ https://hal.science/hal-01361782}

Submitted on 1 Feb 2017

HAL is a multi-disciplinary open access archive for the deposit and dissemination of scientific research documents, whether they are published or not. The documents may come from teaching and research institutions in France or abroad, or from public or private research centers.
L'archive ouverte pluridisciplinaire HAL, est destinée au dépôt et à la diffusion de documents scientifiques de niveau recherche, publiés ou non, émanant des établissements d'enseignement et de recherche français ou étrangers, des laboratoires publics ou privés. 


\section{Static electric field enhancement in nanoscale structures}

Bruno Lepetit' , Didier Lemoine' , and Maykel Márquez-Mijares'

Citation: J. Appl. Phys. 120, 085105 (2016); doi: 10.1063/1.4961216

View online: http://dx.doi.org/10.1063/1.4961216

View Table of Contents: http://aip.scitation.org/toc/jap/120/8

Published by the American Institute of Physics

\section{AlP $\left.\right|_{\text {Applied Physics }} ^{\substack{\text { Journal of } \\ \text { Ans }}}$}

INTRODUCING INVITED PERSPECTIVES

Ultrafast magnetism and THz spintronics

Authors: Jakob Walowski and Markus Münzenberg 


\title{
Static electric field enhancement in nanoscale structures
}

\author{
Bruno Lepetit, ${ }^{1,2, a)}$ Didier Lemoine, ${ }^{1,2, b)}$ and Maykel Márquez-Mijares ${ }^{1,2,3, c)}$ \\ ${ }^{1}$ Université de Toulouse, UPS, Laboratoire Collisions Agrégats Réactivité, IRSAMC, F-31062 Toulouse, \\ France \\ ${ }^{2}$ CNRS, UMR 5589, F-31062 Toulouse, France \\ ${ }^{3}$ Instituto Superior de Tecnologías y Ciencias Aplicadas, Avenida Salvador Allende 1110, \\ Quinta de los Molinos, La Habana, Cuba
}

(Received 9 May 2016; accepted 4 August 2016; published online 29 August 2016)

\begin{abstract}
We study the effect of local atomic- and nano-scale protrusions on field emission and, in particular, on the local field enhancement which plays a key role as known from the Fowler-Nordheim model of electronic emission. We study atomic size defects which consist of right angle steps forming an infinite length staircase on a tungsten surface. This structure is embedded in a $1 \mathrm{GV} / \mathrm{m}$ ambient electrostatic field. We perform calculations based upon density functional theory in order to characterize the total and induced electronic densities as well as the local electrostatic fields taking into account the detailed atomic structure of the metal. We show how the results must be processed to become comparable with those of a simple homogeneous tungsten sheet electrostatic model. We also describe an innovative procedure to extrapolate our results to nanoscale defects of larger sizes, which relies on the microscopic findings to guide, tune, and improve the homogeneous metal model, thus gaining predictive power. Furthermore, we evidence analytical power laws for the field enhancement characterization. The main physics-wise outcome of this analysis is that limited field enhancement is to be expected from atomic- and nano-scale defects. Published by AIP Publishing.

[http://dx.doi.org/10.1063/1.4961216]
\end{abstract}

\section{INTRODUCTION}

Electronic field emission ${ }^{1}$ induced by electrostatic field can result in vacuum breakdown. This poses challenges to the design of vacuum insulation structures in high voltage systems, especially nowadays in the controlled nuclear fusion industry. Indeed, one of the heating strategies of a magnetically confined plasma in tokamaks uses high energy hydrogen or deuterium atoms accelerated by the electrostatic field of a high voltage system, the performance of which is severely limited by damaging electron currents induced by field emission. ${ }^{2-4}$ The intensity of such a field emission current can be reduced by raising the pressure in the vacuum system, typically from high or ultrahigh vacuum to pressures of the order of $10^{-4}-10^{-2} \mathrm{~Pa}^{5-9}$ This effect has been known for quite some time $\mathrm{e}^{10,11}$ and has been investigated recently in detail for tungsten carbide and tungsten cathodes. ${ }^{12-14}$

These changes in field emission intensity with ambient pressure are related to modifications of the cathode surface state at some unknown scale, which may be the micrometer size scale or an even smaller one. There is indeed no consensus on the detailed nature of these surface changes. Some believe that these changes in the emitting properties of the surface result from adsorption of ambient gases or other contaminants. ${ }^{10,15}$ In a recent experimental study, the main contaminant was found to be carbon. ${ }^{14}$ The theoretical work concluded that the presence of carbon contamination increases the work function of tungsten surfaces and hence decreases electronic emission, which is opposite to the

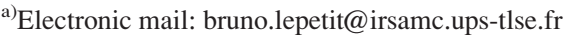

b) Electronic mail: didier.lemoine@irsamc.ups-tlse.fr

${ }^{c}$ Electronic mail: mmarquez@instec.cu
}

experimentally observed trend. ${ }^{16}$ Another mechanism explaining the observed gas effect should therefore be looked for.

Others attribute the gas effect to the transformation of sharp emitting tips into blunt ones by sputtering due to ion bombardment localized near emitting protrusions. ${ }^{6-8}$ Modifying tip morphology may impact field emission in two ways. One of them is through modifications of the local work function. Theoretical studies on the effects of emitting surfaces modifications on local work functions have been performed in Refs. 17-20. Another one is through modifications of the local field enhancement. It is well known ${ }^{5,21}$ that field emission occurs for macroscopic electrostatic field values that are considerably lower than that of the local field values predicted by Fowler-Nordheim theory (see Ref. 1 for the initial theory and Ref. 22 for more recent developments) because the local field enhancement of the macroscopic field reduces the electrostatic potential barrier through which emitted electrons have to tunnel. A local field enhancement factor $\beta$ is defined as the ratio between the local field near a surface emitting site and the macroscopic field. It can be extracted from Fowler-Nordheim plots, provided the emission situation is orthodox as defined in Ref. 23, which should normally be the case for metal emitting surfaces. Such plots are usually close to straight lines, the slopes of which provide the enhancement factors $\beta$ if the work function is known. This enhancement factor can be related to the detailed shape and/or the rugosity of the surface, and there is experimental evidence that smoothing the surface decreases emission. Typically, $\beta$ values of several hundreds have been obtained for an unpolished stainless steel ${ }^{24}$ or copper $^{25}$ surfaces, whereas $\beta$ of several tens have been measured for titanium, molybdenium, ${ }^{26}$ and niobium, ${ }^{27}$ for rugosities of the 
order of ten nanometers or more. Notice however that the connection between enhancement and rugosity is somewhat loose: for instance, higher field enhancement is obtained for reduced rugosity in Ref. 28. This may be related to a lack of control of the emitting surface state at the atomic scale which we define as being of the order of $1 \mathrm{~nm}$ or less and which is a smaller scale than the one usually considered. If atomic scale protrusion is a significant contributor to field enhancement, then control of the rugosity with standard surface treatment may not be enough to obtain reproducible emission. Finding out at which scale the surface state needs to be controlled for reproducible emission is the main objective of the paper.

The role of protrusions on electrostatic field and emission enhancement has already been considered from a theoretical point of view (for a review, see, for instance, Ref. 29). Typically, an order of magnitude of the $\beta$ factors obtained for different shapes of protrusions is given by $\beta \approx \frac{D}{r}$, where $D$ is the height of the emitter tip above the electrode plane which plays the role of a ground plane and $r$ its apex radius of curvature. Beyond this simple scaling law, more accurate results can be obtained by solving the Poisson equation with analytical or numerical methods where the metallic electrodes play the role of boundary conditions for the electrostatic field. ${ }^{29}$ Within such a model, the electrode is considered at the macroscopic scale as an homogeneous metal without consideration of its atomic structure. We refer to such a classical conductor model as Homogeneous Metal Model (HMM) in the following. Yet, the use of HMM for atomic size protrusions is obviously questionable and requires care. At this scale, it is expected that quantum mechanical methods taking into account the atomic structure of the emitting electrode must be used to obtain realistic electronic distributions. Besides, as the use of HMM remains desirable because of its simplicity, careful thought must be given to how to implement it to minimize errors and to gain predictive power. In particular, how should be defined the radius of curvature $r$ of the protrusion tip, which can consist of a single atom in the limiting case? All of these issues are addressed in the present study.

The present paper is organized as follows. In Section II, we describe the protrusion model which we consider to study the local enhancement of an applied external field. We explain how we implement the chosen quantum mechanical method, namely, Density Functional Theory (DFT), to study this problem. Our work is thus one of the very few studies ${ }^{20,30,31}$ on electrostatic field interactions with conductors based on a realistic quantum mechanical description of the material at the atomic scale. Section III is split into 3 parts. First, we analyze the three-dimensional electronic density and electrostatic field distributions resulting from the DFT calculations. Second, we compare these results with those of a standard electrostatic HMM, where the structure is described by its geometry without consideration of its atomic structure, as is done routinely in engineering applications. ${ }^{29}$ This allows to show how the HMM can be implemented to provide results in somewhat surprisingly good agreement with quantum mechanical predictions even in the case of atomic scale protrusions. Third, from these results, we extrapolate conditions which have to be fulfilled for the size and the geometry of a protrusion to achieve a given level of

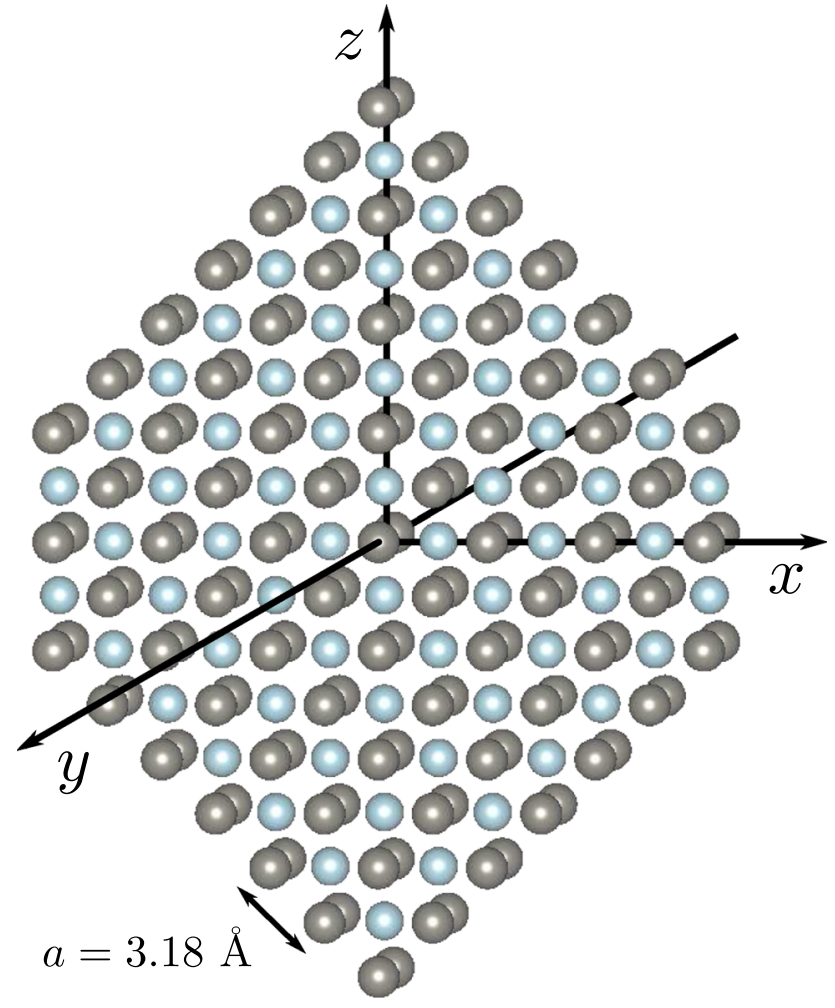

FIG. 1. Unit cell considered in the DFT model of the tungsten step structure. The structure reproduces periodically along the $x$ and $y$ (perpendicular to the figure plane) directions. Three atomic $(x, z)$ planes are shown: the 1st and 3rd ones which are identical by periodicity correspond to the grey atoms, and the 2 nd one to the blue atoms. The size of the bulk cubic unit cell $a=$ $3.18 \AA$ is also shown. $a$ is also the distance between the 1 st and $3 \mathrm{rd}$ atomic planes of the figure.

field enhancement. Section IV provides our conclusions, in particular, on the enhancement of external fields by atomicand nano-scale protrusions.

\section{COMPUTATIONAL METHODS}

\section{A. Structure model}

We consider bcc tungsten as this is a common basic material for electrodes which we have already considered in a previous study of the effect of carbon adsorption on flat (100) surfaces. ${ }^{16}$ We know from this previous study that the lattice parameter is $a=3.18 \AA$. We now consider a tungsten surface with defects which consist of right angle steps of width and height $6 a$ parallel to the (100) and (010) planes. Such steps are common surface defects at the atomic scale. A single step is shown on the upper half of Fig. 1 with the $x$ and $z$ axes tilted at $45^{\circ}$ with respect to the step planes. A symmetric structure is generated by reflection through the $(x, y)$ plane. This structure is the unit cell of our calculation. It consists in two $(x, z)$ atomic planes separated by a distance $a / 2=1.59 \AA$ along the $y$ direction. It contains a total of 132 atoms, 72 in the first plane and 60 in the second. By repeating periodically this unit cell in the $y$ direction, the step length becomes infinite along the $y$ direction. Periodic extension along the $x$ direction generates an infinite staircase made of square steps. The final structure can also be viewed as consisting of triangular parallel ridges, or equivalently of two lines of an infinite thickness (along $y$ ) right angle saw 
teeth. The upper line describes defects on the cathode, the lower one on the anode, and they reflect each other through the $x, y$ mid-plane. The teeth tips and the troughs between them are distant (along $z$ ) from this mid-plane by $4 \sqrt{2} a=$ 18. $\AA$ and $\sqrt{2} a=4.5 \AA$, respectively. On a given side of the mid-plane, the lateral distance (along $x$ ) between neighboring tips is $6 \sqrt{2} a=27 . \AA$.

This unit cell is enclosed in a box including vacuum on both sides of the structure, which is $117 \AA$ long in the $z$ direction. As we use a periodic DFT method, the structure is replicated periodically in the $z$ direction and the box has to be large enough to avoid electrostatic interactions between replicates. This box size also complies with the rule, ${ }^{32}$ which states that the distance between the electrode and the counter electrode (here $117 \AA$ ) must be several times larger than that of the height of the field-enhancing structure (here $18 . \AA$ ) to avoid the distribution of field near this structure to be significantly affected by the proximity of the counter-electrode. Both sides of this structure are embedded in a static far field $\mathrm{E}_{\infty}$ pointing along the negative $z$ direction so that the upper and lower step sides play the role of a cathode and of an anode, respectively. The cathode-anode separation through the thickness of the structure varies between $2 \sqrt{2} a=9 \AA$ and $8 \sqrt{2} a=36 \AA$ at the tip. Since anode and cathode are thus well separated, they can be considered as noninteracting between each other. We use this stepped structure as a simple model for protrusions, and we obtain results simultaneously for anode and cathode configurations.

\section{B. DFT computations}

The $a b$ initio total-energy and molecular dynamics program Vienna $a b$ initio simulation program (VASP) developed at the Institut für Materialphysik of the Universität Wien has been used for all DFT calculations. ${ }^{33-36}$ We use this program in a way very similar to the one already described in Ref. 16. The electron-ion interaction for tungsten is described by the projector augmented wave potential (PAW). ${ }^{37,38}$ The exchange-correlation energy is calculated within the generalized gradient approximation (GGA) using the revised form of the Perdew, Burke, and Ernzerhof functional (PBE). ${ }^{39-41}$ We consider six $5 d 6 s$ valence electrons for each tungsten atom, and we use the convergence parameters resulting from a previous study. ${ }^{16}$ Fractional occupancies are calculated using a second-order Methfessel-Paxton smearing function ${ }^{42}$ with a width of $0.2 \mathrm{eV}$. All plane waves of the basis set are expanded up to a kinetic energy cutoff of $580 \mathrm{eV}$, ensuring a good convergence of total energies with an accuracy of the order of $5 \mathrm{meV}$. We use a $(5 \times 11 \times 1) \mathrm{k}$ point grid, and we checked convergence in comparison with $(7 \times 11 \times 1)$ and $(5 \times 15 \times 1)$ grids. We do not allow for geometry relaxation of the tungsten structure in our study. Not only this simplification maintains computing times within reasonable limits but also it is furthermore necessary in order to compare our quantum mechanical results with HMM ones performed for undistorted geometric shapes.

The DFT step model is embedded in an external electrostatic field $\mathrm{E}_{\infty}=0.1 \mathrm{~V} / \AA$. This is strong in comparison with macroscopic fields likely to be met in engineering applications but enables operating local field values (typically $0.1-1 \mathrm{~V} / \AA$ ) to be achieved with field enhancement factors of modest size. In the presence of this external field, an artificial dipole must be added in the vacuum region to allow for periodicity of the electrostatic potential between the upper and lower rim of the supercell. This correction also cancels the long range dipolar interactions between the periodic replicates of the structure in the $z$ direction.

The DFT calculations provide 3-dimensional (3D) quantities like the charge density $\rho(x, y, z)$ and the electrostatic potential $V(x, y, z)$, from which the electrostatic field $\mathbf{E}(x, y, z)$ can be easily derived. These quantities oscillate strongly as a function of the position especially near the atoms forming the metallic structure and are not well suited for direct comparison with HMM results. Indeed, the HMM quantities are smooth ones, and HMM charge density and electrostatic field are null inside the structure. Following Fall et al., ${ }^{17-19}$ we define locally averaged quantities labelled with bars, for instance: $\bar{\rho}(x, y, z)=\iiint_{v(x, y, z)} d x^{\prime} d y^{\prime} d z^{\prime}$ $\rho\left(x^{\prime}, y^{\prime}, z^{\prime}\right) / v(x, y, z)$. The integration is performed on a $\left[\frac{a}{\sqrt{2}}, a, \frac{a}{\sqrt{2}}\right]$ rectangular cuboid $v(x, y, z)$ centered around the point $(x, y, z)$. Locally averaged quantities do have the same behaviour as the HMM ones inside the bulk of the material (zero total charge and field). With our particular choice of average length in the $y$ direction, equal to the periodicity $a$, the locally averaged quantities are independent of $y$ and will be represented in the following as two-dimensional $x, z$ quantities. Derivations and averaging of the 3D quantities have been performed by using Fourier transforms.

\section{RESULTS}

\section{A. DFT results}

Figure 2 (left) shows the locally averaged electronic density $\bar{\rho}\left(\mathrm{E}_{\infty}=1 \mathrm{GV} / \mathrm{m}\right)$ around the structure embedded in the external field $E_{\infty}=1 \mathrm{GV} / \mathrm{m}$. This figure is undistinguishable from the one obtained without external field. This density is constant inside the bulk, and its value $\bar{\rho}_{0}=$ $0.37 \mathrm{e} / \AA^{3}$ (e: electron charge) which compensates the constant locally averaged tungsten nuclei positive charge $(6$ charges per atom, 2 atoms per cubic unit cell, $a=3.18 \AA$ ) and provides global electrical neutrality. Using a ThomasFermi model, it is possible to show that the density decreases from the bulk value to zero over a distance of the order of the Thomas-Fermi screening length given by (Ref. 43, p. 281): $\left.\lambda_{s} \approx \frac{\pi^{\frac{1}{6}}}{23^{\frac{1}{6}}} \frac{a_{0}^{3}}{\bar{\rho}_{0}}\right)^{\frac{1}{6}}$, where $a_{0}$ is the Bohr radius, which gives in the present case: $\lambda_{s} \approx 0.42 \AA$. As confirmed by the more exact theory of Lang and Kohn, ${ }^{44,45}$ this smooth decrease is associated with an excess of a net (i.e., including nuclei charges) positive charge at the interface on the bulk side and an excess of negative charge on the vacuum side. This electric dipolar sheet generates a surface electrostatic field pointing toward the vacuum as shown in Fig. 3 (left). This field which can be as high as $6.5 \mathrm{~V} / \AA$ in the present case contributes to the confinement of the metal electron gas.

The perturbation associated with the external electrostatic field $\mathrm{E}_{\infty}=1 \mathrm{GV} / \mathrm{m}$ on $\bar{\rho}$ is very small. Indeed, Fig. 2 (right) shows that the difference between the 2 electronic 

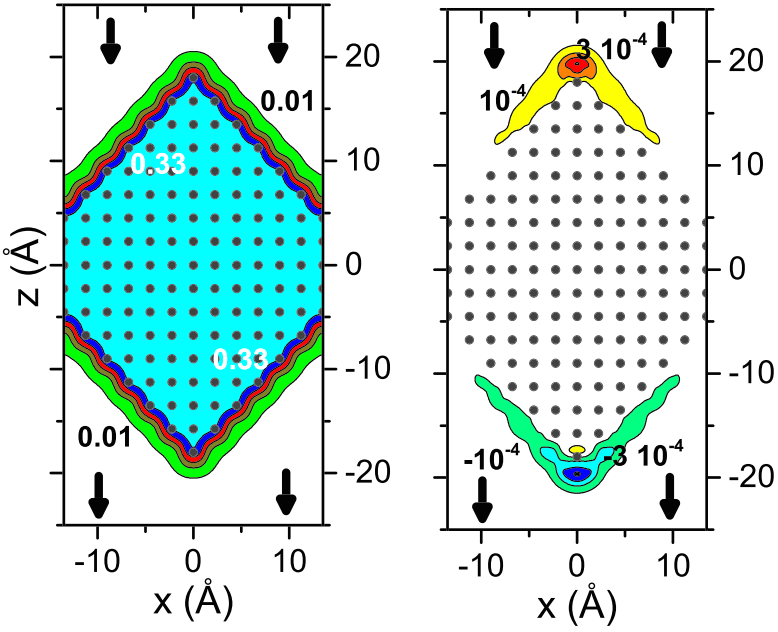

FIG. 2. Left: locally averaged electronic charge density $\bar{\rho}\left(\mathrm{E}_{\infty}=0.1 \mathrm{~V} / \AA\right)$ in e $/ \AA^{3}$. As the averaging is performed on the periodicity of the structure $a$ in the $y$ direction, the locally averaged charge density is independent of $y$. The structure is subjected to the $\mathbf{E}_{\infty}=0.1 \mathrm{~V} / \AA$ far field parallel to the $z$ axis and symbolized by the pairs of vertical arrows on both sides of the metallic structure. The contours range from $0.01 \mathrm{e} / \AA^{3}$ to $0.33 \mathrm{e} / \AA^{3}$ and are equally spaced by $0.08 \mathrm{e} / \AA^{3}$. The dots indicate the locations of the tungsten atoms. The constant value of the locally averaged electronic density in the bulk of the metal is $0.37 \mathrm{e} / \AA^{3}$. Right: induced locally averaged electronic charge $\bar{\rho}\left(\mathrm{E}_{\infty}=0.1 \mathrm{~V} / \AA\right)-\bar{\rho}\left(\mathrm{E}_{\infty}=0.0 \mathrm{~V} / \AA\right)$ resulting from the effect of the vertical, downward external field $\mathrm{E}_{\infty}=0.1 \mathrm{~V} / \AA$ (vertical arrows). The contours range from $-7 \times 10^{-4} \mathrm{e} / \AA^{3}$ to $7 \times 10^{-4} \mathrm{e} / \AA^{3}$ and are equally spaced by $2 \times 10^{-4} \mathrm{e} / \AA^{3}$. As expected, there is an excess charge density with a maximum value of $7.1 \mathrm{e} / \AA^{3}$ near the tip on the upper (cathode) side, and a deficit with a maximum absolute value $7.25 \mathrm{e} / \AA^{3}$ near the tip on the lower (anode) side.

charge distributions, with and without external field $\bar{\rho}\left(\mathrm{E}_{\infty}=0.1 \mathrm{~V} / \AA\right)-\bar{\rho}\left(\mathrm{E}_{\infty}=0.0 \mathrm{~V} / \AA\right)$, is typically 3 orders of magnitude smaller than that of the electronic charge distribution $\bar{\rho}$ itself. As expected, there is an excess of electrons on the cathode side and a deficit on the anode side. These induced charges concentrate mainly close to the tips of the structure. Interestingly, the induced charge is not localized on the top most atomic planes but is shifted toward the vacuum. We define the Induced Charge Barycenter (ICB) by

$$
z_{I C B}\left(x, y, \mathrm{E}_{\infty}\right)=\frac{\int z\left(\bar{\rho}\left(x, y, z, \mathrm{E}_{\infty}\right)-\bar{\rho}(x, y, z, 0)\right) d z}{\int\left(\bar{\rho}\left(x, y, z, \mathrm{E}_{\infty}\right)-\bar{\rho}(x, y, z, 0)\right) d z},
$$

and we call $z_{t i p}= \pm 18 \AA$ the height of the atom at the tip of the cathode or anode steps. This definition is similar to the one proposed in Ref. 45 in the context of the modelling of flat metallic surfaces in the jellium picture. We can easily compute the shift of the induced charge with respect to the tip, and we find: $\left|z_{I C B}\left(x_{t i p}, y_{t i p}, \mathrm{E}_{\infty}=1 \mathrm{GV} / \mathrm{m}\right)-z_{t i p}\right|=1.61$ and $2.13 \AA$ on the anode and cathode sides, respectively. This shift is equivalent to the "repulsion distance" used in the charged surfaces theory in field ion emission contexts. ${ }^{46,47}$ For flat tungsten (110), this repulsion distance was calculated in Ref. 47 to be $157 \mathrm{pm}$, which is close to the present results.

Figure 3 (left) shows the norm of the locally averaged electrostatic field around the metallic structure in the presence of the external field. The maximum intensity near the surface is $6.5 \mathrm{~V} / \AA$, much larger than that of the asymptotic value $\mathrm{E}_{\infty}=0.1 \mathrm{~V} / \AA$. Let us consider the field along the

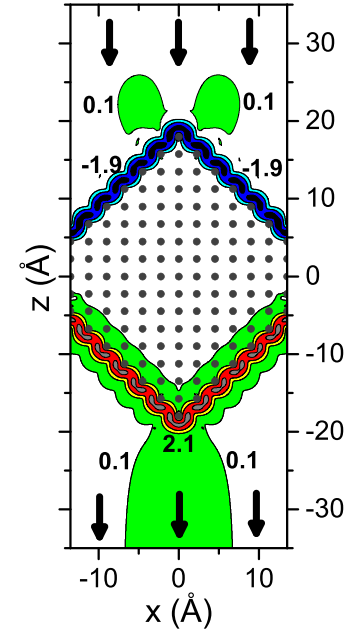

(a)

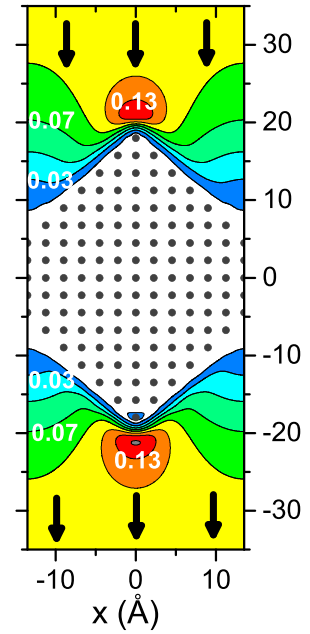

(b)
FIG. 3. Left: norm of the locally averaged electrostatic field $\mid \overline{\mathbf{E}}\left(\mathrm{E}_{\infty}\right.$ $=0.1 \mathrm{~V} / \AA) \mid$ multiplied by the sign of its $z$ component. As the averaging is performed on the periodicity of the structure $a$ in the $y$ direction, the locally averaged electrostatic field is independent of $y$. Downward field is positive. The contours range from $-5.9 \mathrm{~V} / \AA$ to $6.1 \mathrm{~V} / \AA$ and are equally spaced by 2 $\mathrm{V} / \AA$. . The far electrostatic field $\mathbf{E}_{\infty}$ symbolized by the triplets of vertical arrows is the same on both sides of the metallic structure; it is parallel to the $z$ axis, with an amplitude of $0.1 \mathrm{~V} / \AA$. The dots indicate the locations of the tungsten atoms. On the upper side of the metallic structure (cathode side), the field is pointing upward (negative contour values), in the opposite direction to that of the applied field. On the lower side (anode), the field is pointing downward. Right: norm of the induced field, defined as the difference of the electrostatic fields with and without asymptotic field $\mid \overline{\mathbf{E}}\left(\mathrm{E}_{\infty}=0.1 \mathrm{~V} / \AA\right)$ $-\overline{\mathbf{E}}\left(\mathrm{E}_{\infty}=0 \mathrm{~V} / \AA\right) \mid$. The contours now range from $-0.01 \mathrm{~V} / \AA$ to $1.5 \mathrm{~V} / \AA$ and are equally spaced by $0.02 \mathrm{~V} / \AA$.

vertical line passing through the tips (fixed $\left.x_{t i p}, y_{t i p}\right)$. By symmetry, along this line, only the $z$ component of the field is nonzero. By convention, we choose positive values for this component when the field is pointing downward. On the anode side, the field $z$ component increases uniformly from its positive asymptotic value to its maximum $6.5 \mathrm{~V} / \AA$ at a distance $\left|z_{\mathrm{E} \max }-z_{\text {tip }}\right|=1.2 \AA$ from the tip-atom nucleus. On the contrary, on the cathode side, the field $z$ component decreases from its asymptotic value, changes sign at $\left|z_{0}-z_{t i p}\right|=3.8 \AA$, and reaches a minimum $-6.5 \mathrm{~V} / \AA$ at a distance $\left|z_{\mathrm{E} \text { min }}-z_{\text {tip }}\right|=1.1 \AA$. Therefore, the anode and the cathode do not behave similarly with respect to the external field, and instead of a field enhancement on the cathode side, a field inversion is observed.

Figure 3 (right) shows the norm of the difference of the electrostatic fields with and without asymptotic field: $\Delta \mathrm{E}=\left|\overline{\mathbf{E}}\left(\mathrm{E}_{\infty}=0.1 \mathrm{~V} / \AA\right)-\overline{\mathbf{E}}\left(\mathrm{E}_{\infty}=0 \mathrm{~V} / \AA\right)\right| . \Delta \mathrm{E}$ is generated by the induced charge of Fig. 2 (right). At the vertical of the tips, $\Delta \mathrm{E}$ has a maximum on both sides of the structure $\Delta \mathrm{E}=0.15 \mathrm{~V} / \AA$ for: $\left|z_{\Delta \mathrm{Emax}}-z_{\text {tip }}\right| \approx 3.3 \AA$. We show in Section III B that this maximum can be related to the usual electrostatic field enhancement occurring in the vicinity of metallic tips.

\section{B. Comparison with HMM results}

In the HMM, the tungsten step is described as an homogeneous metal, without consideration of the atomic scale structure. The geometry considered and shown in Fig. 4 is a 


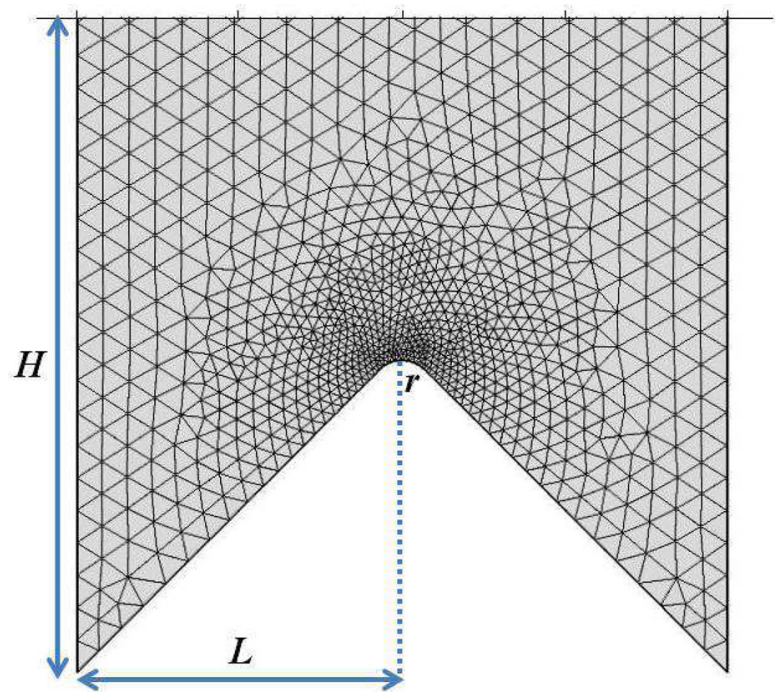

FIG. 4. Example of a mesh used in the Homogeneous Metal Model (HMM). The meshed part is the vacuum, and periodic boundary conditions are applied on the left and right sides. Perfect metal boundary conditions are applied on the upper side, set at some arbitrary nonzero potential, and on the step contour, set at zero potential. Also shown is the step size $L$, the vacuum thickness $H$, and the radius of curvature $r$ of the tip.

right angle step of finite size $L$ terminated by a tip with a radius of curvature $r$. This 2D step can also be considered as a $3 \mathrm{D}$ one extending infinitely in the direction perpendicular to the plane of the figure. The meshed area corresponds to the vacuum between the step at the bottom and a metallic plate at height $H$. Fields and charges are computed with a commercial electrostatic solver for the ambient field $\mathrm{E}_{\infty}=$ $1 \mathrm{GV} / \mathrm{m}$ already considered for the DFT model. The step contour is at zero potential, and the upper plate is at the appropriate potential to generate this ambient electrostatic field. As previously, the step is periodically replicated laterally to generate an infinite staircase by implementing appropriate periodic boundary conditions on the left and right sides of the meshed area. This HMM corresponds to one of the two step configurations, the anode and the cathode, considered simultaneously in the DFT model of Section III A. Indeed, it is enough to consider a single configuration in the HMM, where the anode and the cathode are fully symmetric, whereas they are not in the DFT, as described in Section III A.

The geometry considered in the DFT calculation provides $L=13.5 \AA$ in the HMM, but it does not give indications on what should be the appropriate value for the tip radius $r$ to obtain HMM results in good agreement with DFT ones. It is therefore necessary to test different $r$ values, compute HMM charges and electrostatic fields, and compare them with the DFT results of Section III A. After a few iterations, we found that $r=1.62 \AA$ provides HMM results in good agreement with DFT ones. This is a typical atomic radius value for tungsten, for instance, we used the atomic radius $r_{a}=1.38 \AA$ in Ref. 16 in a different context. Finally, the height $H$ is not a critical parameter, it has to be taken large enough so that the field near the upper plate is uniform and small enough for the calculation to be tractable, $H=$ $135 \AA$ was found to be a good compromise. The mesh of this

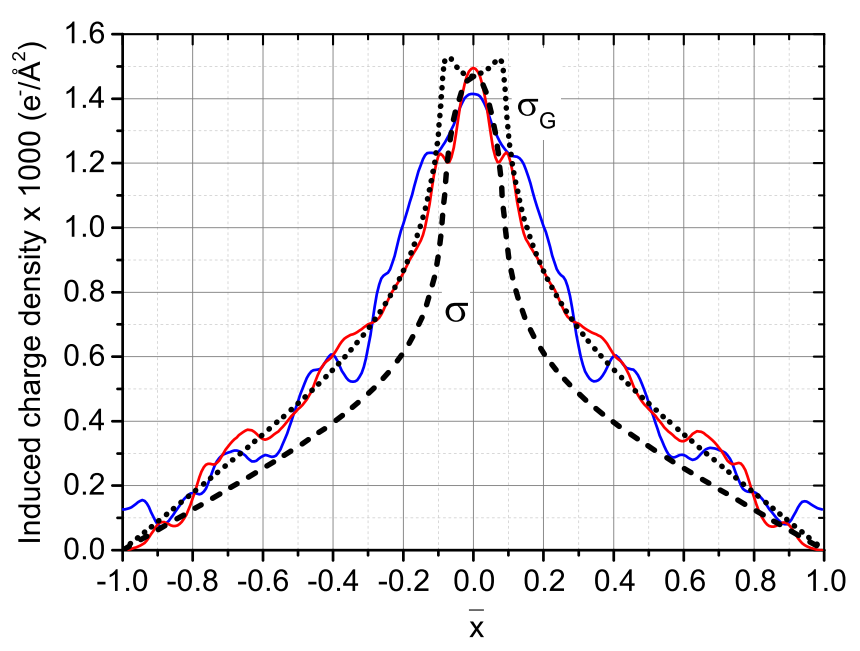

FIG. 5. Absolute value of the induced surface charge as a function of the reduced abscissa $\bar{x}=x / L$ ( $x$ as in Figs. 2 or 3). Full lines: difference between the locally averaged charge distributions resulting from the DFT model for the applied external fields $E_{\infty}=0.1 \mathrm{~V} / \AA$ and $E_{\infty}=0.0 \mathrm{~V} / \AA$ after integration along the field direction $z:\left|\int d z\left(\bar{\rho}\left(x, y, z, \mathrm{E}_{\infty}\right)-\bar{\rho}(x, y, z, 0)\right)\right|$. Anode (blue) and cathode (red) cases correspond to integration on complementary sides with respect to the mid-plane of the metallic structure for $z<0$ and $z>0$, respectively. Dashed line: surface charge $\sigma$ resulting from the electrostatic HMM, with $\mathrm{E}_{\infty}=0.1 \mathrm{~V} / \AA$ and $r=1.62 \AA, L=13.5 \AA, H=135 \AA$. Dotted line: modified induced surface charge $\sigma_{G}=\sigma / \cos \theta_{s}$, where $\theta_{s}$ is the angle between the normal to the surface and the vertical axis $z$.

geometry involving a significant refinement near the tip where charges and fields are strongly dependent on position corresponds to 5524 domain elements and 268 frontier elements.

Figure 5 shows the HMM surface charge density as a function of the reduced abscissa $\bar{x}=x / L$. As expected, charge density is symmetric and largest at the tip location $\bar{x}=0$. In order to compare with DFT volumic charge density, integration is performed over the thickness of the material, and we define an induced DFT surface charge: $\sigma_{D F T}(x, y)=\left|\int d z\left(\bar{\rho}\left(x, y, z, \mathrm{E}_{\infty}\right)-\bar{\rho}(x, y, z, 0)\right)\right|$, where the integrand is the induced volumic charge difference already shown in Fig. 2 (right). Integration is performed for $z<0$ and $z>0$ for the cathode and anode cases, respectively. Apart from the faint oscillations reminiscent of the atomic structure, the cathode and anode DFT integrated densities are close to each other, as shown in Fig. 5. They however differ somewhat from the HMM surface density. Agreement can be improved if a geometrical factor is taken into account. Indeed, if the HMM provided a volumic charge density, surface density would be obtained by integrating along the normal to the surface, and not along the $z$ direction as is done with the DFT volume distribution. Such a $z$ integration can be straightforwardly mimicked in the HMM by considering $\sigma_{G}=\sigma / \cos \theta_{s}$, where $\theta_{s}$ is the angle between the normal to the surface and the $z$ axis. This HMM surface charge density with geometrical correction is then much closer to the DFT induced integrated charge density, as shown in Fig. 5.

Figure 6 compares the DFT averaged induced field (the same as the one already shown in the $2 \mathrm{D}$ plot of Fig. 3 (right)) with the HMM electrostatic field. The best agreement is obtained when the metallic surface is chosen, not as the top most atomic planes, but as the planes defined by the 


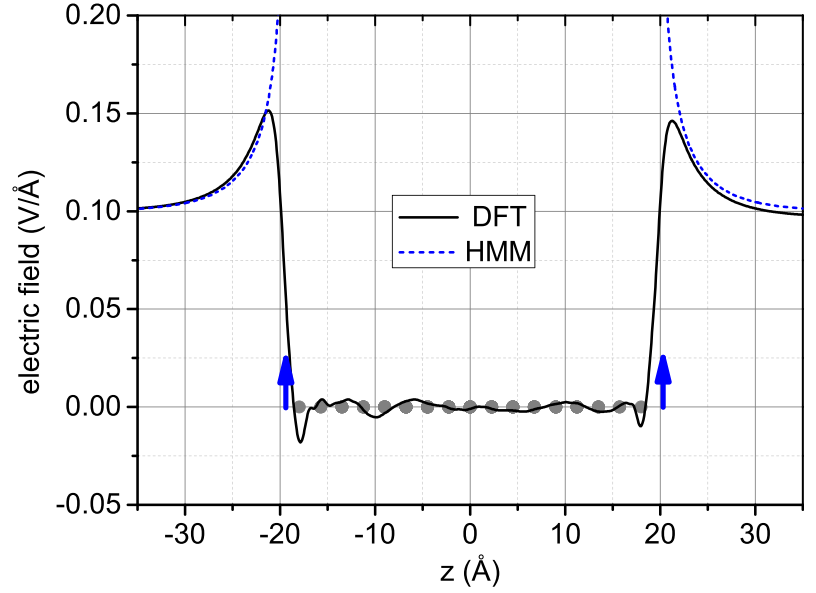

FIG. 6. Full line: Density functional theory (DFT) result: difference $\left(\mathrm{E}_{z}\left(\mathrm{E}_{\infty}=0.0 \mathrm{~V} / \AA\right)-\mathrm{E}_{z}\left(\mathrm{E}_{\infty}=0.1 \mathrm{~V} / \AA\right)\right)$ between locally averaged, $z$ component electrostatic fields corresponding to $\mathrm{E}_{\infty}=0.0 \mathrm{~V} / \AA$ and $\mathrm{E}_{\infty}=0.1 \mathrm{~V} / \AA$, along a line perpendicular to the slab passing through the tip ( $z$ axis on Fig. 1). Dashed line: Electrostatic field resulting from the electrostatic HMM with $r=1.62 \AA, L=13.5 \AA, H=135 \AA$. The conductor planes used in the HMM do not coincide with the atomic planes, but with the Induced Charge (shown on Fig. 2) Barycenter (ICB) planes (see text, Eq. (1)). These ICB plane positions are symbolized by the vertical blue arrows. The resulting shifts with respect to the atom locations are $2.13 \AA$ on the cathode side $(z>0$ on the figure) and $1.61 \AA$ on the anode side $(z<0)$. The HMM electrostatic field at these locations is $0.26 \mathrm{~V} / \AA$. Grey dots: atomic plane locations.

induced charge barycenter $z_{I C B}\left(x, y, \mathrm{E}_{\infty}\right)$ (see Eq. (1)). The shifts with respect to the atom location planes are $2.13 \AA$ on the cathode side and $1.61 \AA$ on the anode side $(z<0)$. The maximum HMM electrostatic field at these locations is $0.26 \mathrm{~V} / \AA$, which corresponds to a field enhancement factor $\beta=2.6$ only. The DFT maximum induced field is smaller

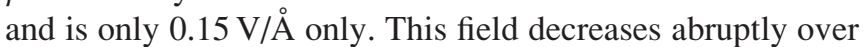
a distance of $2 \AA$ or so inside the volumic charge distribution near the surface which screens the external field.

As a conclusion of this section, we have shown that a simple HMM is capable of reproducing charge and field distributions at the atomic scale if they are appropriately processed. Induced charges and fields have to be extracted from raw DFT results and subject to averaging and integration procedures. An appropriate radius of curvature must be chosen for the tips in the HMM. For the present right angle step, we found that the right angle must be represented by a radius of curvature of the order of the atomic radius of the tip atom. Finally, we found in both models a modest field enhancement of the order of a factor of 2 .

\section{Extrapolation to other defect sizes}

We now study what should be the appropriate size range for the defect considered (right angle step) to yield a given local field enhancement $\beta$. For the atomic size step considered up to now, an enhancement is small. What should be the conditions for larger enhancement? This study is most likely not feasible within the DFT framework due to computer time limitation, but it is straightforward with HMM using an electrostatic model, once the relation between HMM and DFT results is established according to the results of Section III B.

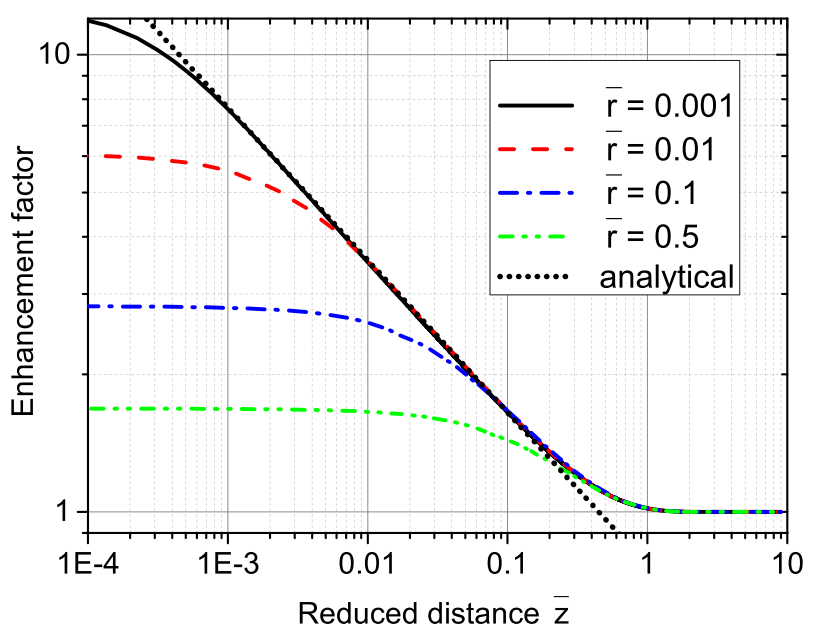

FIG. 7. Numerical field enhancement $\beta(\bar{z}, \bar{r})$ as a function of the reduced distance: $\bar{z}=\frac{z-z_{\text {tip }}}{L}$ for different reduced radii $\bar{r}=\frac{r}{L}$. The dotted line represents the function: $\beta^{a}(\bar{z})=\left(\frac{9}{20 \bar{z}}\right)^{\frac{1}{3}}$, which fits closely $\beta(\bar{z}, \bar{r})$ in the intermediate distance region $\bar{r}<\bar{z} \ll 1$ where the field enhancement is independent of $\bar{r}$. The HMM situation depicted in Fig. 6 corresponds to $\bar{r}=0.12$ and $\beta=2.6$, close to the blue curve $(\bar{r}=0.1)$.

We first notice that among the 3 geometrical parameters $r, L$, and $H$ in the HMM, $H$ can be ignored as it is a mere numerical convergence parameter not related to the physics of the problem. Simply, $H$ must be chosen large enough to ensure that the structure is embedded in a uniform far field. Then, one should notice that the problem can be made independent of $L$ by using reduced coordinates: $\bar{r}=r / L$ and $\bar{z}=\left(z-z_{\text {tip }}\right) / L$. Fig. 7 shows the enhancement factor $\beta(\bar{r}, \bar{z})$ as a function of the reduced distance at the vertical of the tip for different geometries labelled by $\bar{r}$. In the interval $r<z-z_{\text {tip }} \ll L$, the relation between the enhancement factor and the reduced distance can be fitted with a simple power law $\beta^{a}(\bar{z})$

$$
\beta(\bar{r}, \bar{z}) \approx \beta^{a}(\bar{z})=\left(\frac{9}{20 \bar{z}}\right)^{\frac{1}{3}}
$$

This power law is reminiscent of the infinite step case. Indeed, the electrostatic field and charge distributions associated to a two-dimensional right angle metallic step of infinite length (similar to that of Fig. 4 but assuming $r=0$ and $L \rightarrow \infty$ ) can be easily obtained analytically through a conformal map (Ref. 48, p. 298). The field at the vertical of this infinite tip is given by the power law: $\mathrm{E}_{z} \propto\left(z-z_{\text {tip }}\right)^{-\frac{1}{3}}$. Similarly, the charge per unit surface $\sigma$ on the metal at a distance $\delta$ from the apex is: $\sigma \propto \delta^{-\frac{1}{3}}$. Both field and density thus diverge at the tip, in contrast with the DFT and HMM results for finite $L$. But according to Eq. (2), we find numerically the same $(-1 / 3)$ power law at finite $L$. This law is universal, $\beta^{a}(\bar{z})$ being independent if $\bar{r}$ for $r<z \ll L$, as expected far enough from the tip where apex details have no significant influence.

On the other hand, when $z \ll r$, the enhancement becomes independent of $z$, the dependence of this constant value with respect to $\bar{r}$ can be fitted again accurately by a power law 


$$
\beta(\bar{r}, \bar{z}=0) \approx\left(\frac{9}{4 \bar{r}}\right)^{\frac{1}{3}}=\beta^{a}(\bar{r} / 5) .
$$

The above equation can be used to guess the appropriate step size for some target enhancement $\beta$. This equation indeed provides the appropriate $\bar{r}$. From Section III B, we know that the radius of curvature at the apex should be close to an atomic radius: $r \approx r_{a}$. Hence, an appropriate step size for some target enhancement is given by

$$
L(\beta) \approx r_{a} \frac{4 \beta^{3}}{9} .
$$

As a consequence of this cubic law, large enhancement factors require large step sizes. For instance, $\beta=20$ requires micrometer-size steps: $L \approx 0.6 \mu \mathrm{m}$. A large enhancement cannot be achieved by atomic scale steps at right angle $\alpha=\pi / 2$. This rule is presumably generalizable to steps with any tip angle value $\alpha$. Following again Ref. 48, p. 297, we know that the analytical field dependence with respect to the distance from an infinite step with tip angle $\alpha$ is given by the power law: $\mathrm{E}_{z} \propto\left(z-z_{t i p}\right)^{-\frac{\pi-\alpha}{2 \pi-\alpha}}$. The appropriate step size yielding $\beta$ enhancement becomes: $L(\beta) \propto \beta^{\frac{2 \pi-\alpha}{\pi-\alpha}}$, the smallest step being obtained for the limiting and unrealistic case $\alpha=0$, yielding $L(\beta) \propto \beta^{2}$. Even in this latter case, the square law provides a minimum step size which increases quickly with target enhancement factor. Better enhancement factors $^{49}$ can be achieved if cylindrically symmetric geometries (around $z$ ) like rounded whiskers are used instead of the translationally invariant (along $y$ ) ridges considered here, even for $\alpha=0$.

\section{CONCLUSION}

We studied the effect of local atomic- and nano-scale protrusions on field emission and, in particular, on the local field enhancement which plays a key role as shown by the Fowler-Nordheim model of emission. We focused on model local defects which consist of right angle steps aligned as an infinite length staircase on a tungsten surface. This structure was embedded in a strong external electrostatic field. We performed calculations based upon the density functional theory in order to characterize the total and induced electronic densities as well as the local electrostatic fields, thereby taking into account the atomic structure of the metal. We showed how DFT results could be transformed in order to be compared with those stemming from a simple electrostatic homogeneous metal model. Thus, we achieved somewhat surprisingly good agreement. From that transformation, we also described a procedure to extrapolate our results to defects at different size scales. Hence and qualitatively, we not only validated the homogeneous metal model against the microscopic one but also used the DFT findings to guide, tune, and improve the HMM which in turn gained predictive power. Furthermore, we evidenced analytical power laws for the field enhancement characterization. The main physicswise outcome of this analysis is that limited field enhancement is to be expected from atomic- and nano-size scale defects and that the current polishing procedures for emitting surfaces, where rugosity can be of the order of $10 \mathrm{~nm}$ but is often larger, are good enough to provide field enhancement control. In perspective, our innovative strategy combining the microscopic and macroscopic points of view enables highly efficient nanoscale modelling while preserving a good level of accuracy. It can obviously be applied to investigate other kinds of nanoscale protrusions and defects, including impurities and vacancies. It should also prove useful for engineering applications.

\section{ACKNOWLEDGMENTS}

This work was partly funded by Grant No. ANR-12BS09-0013-02. We thank Jean-Marc L'Hermite and Julien Mauchain for stimulating discussions. The COMSOL Multiphysics ${ }^{\circledR}$ software was used to obtain the HMM results. The authors thank one anonymous referee for careful reading and many constructive comments.

${ }^{1}$ R. H. Fowler and L. Nordheim, Proc. R. Soc. London, Ser. A 119, 173 (1928).

${ }^{2}$ A. Simonin, H. D. Esch, L. Doceul, L. Christin, F. Faisse, and F. Villecroze, Fusion Eng. Des. 88, 1 (2013).

${ }^{3}$ R. S. Hemsworth, A. Tanga, and V. Antoni, Rev. Sci. Instrum. 79, $02 \mathrm{C} 109$ (2008).

${ }^{4}$ R. Hemsworth, H. Decamps, J. Graceffa, B. Schunke, M. Tanaka, M. Dremel, A. Tanga, H. P. L. de Esch, F. Geli, J. Milnes et al., Nucl. Fusion 49, 045006 (2009).

${ }^{5}$ R. V. Latham, High Voltage Vacuum Insulation: Basic Concepts and Technological Practice (Elsevier, New York, 1995).

${ }^{6}$ D. Alpert, D. Lee, E. M. Lyman, and H. E. Tomaschke, J. Appl. Phys. 38, 880 (1967).

${ }^{7}$ G. Beukema, Physica 61, 259 (1972).

${ }^{8}$ R. N. Bloomer and B. M. Cox, Vacuum 18, 379 (1968).

${ }^{9}$ B. Bonin, Vacuum 46, 907 (1995).

${ }^{10}$ A. Zeitoun-Fakiris and B. Juttner, J. Phys. D: Appl. Phys. 21, 960 (1988).

${ }^{11}$ Y. Yamamoto and T. Miyokawa, J. Vac. Sci. Technol. B (Microelectron. Nanometer Struct.) 16, 2871 (1998).

${ }^{12} \mathrm{~K}$. Almousa Almaksour, Ph.D. thesis (Paris-Sud University, 2014).

${ }^{13}$ K. Almaksour, M. J. Kirkpatrick, P. Dessante, E. Odic, A. Simonin, H. P. L. de Esch, B. Lepetit, D. Alamarguy, F. Bayle, and P. Teste, Phys. Rev. Spec. Top. Accel. Beams 17, 103502 (2014).

${ }^{14}$ M. Márquez-Mijares, B. Lepetit, D. Lemoine, K. Almaksour, M. J. Kirkpatrick, P. Dessante, E. Odic, D. Alamarguy, F. Bayle, P. Teste et al., J. Vac. Sci. Technol. (to be published).

${ }^{15}$ R. Gomer and J. K. Hulm, J. Chem. Phys. 27, 1363 (1957).

${ }^{16}$ M. Márquez-Mijares, B. Lepetit, and D. Lemoine, Surf. Sci. 645, 56 (2016).

${ }^{17}$ C. J. Fall, N. Binggeli, and A. Baldereschi, Phys. Rev. B 58, R7544 (1998).

${ }^{18}$ C. J. Fall, N. Binggeli, and A. Baldereschi, Phys. Rev. B 66, 075405 (2002).

${ }^{19}$ C. J. Fall, N. Binggeli, and A. Baldereschi, Phys. Rev. Lett. 88, 156802 (2002).

${ }^{20}$ F. Djurabekova, A. Ruzibaev, E. Holmström, S. Parviainen, and M. Hakala, J. Appl. Phys. 114, 243302 (2013).

${ }^{21}$ R. V. Latham, High Voltage Vacuum Insulation: A New Perspective (AuthorHouse, 2006).

${ }^{22}$ R. G. Forbes, J. H. B. Deane, A. Fischer, and M. S. Mousa, Jordan J. Phys. 8, 125 (2015).

${ }^{23}$ R. G. Forbes, Proc. R. Soc. London, Ser. A 469, 20130271 (2013).

${ }^{24}$ B. M. Cox and W. T. Williams, J. Phys. D: Appl. Phys. 10, L5 (1977).

${ }^{25}$ C. Suzuki, T. Nakanishi, S. Okumi, T. Gotou, K. Togawa, F. Furuta, K. Wada, T. Nishitani, M. Yamamoto, J. Watanabe et al., Nucl. Instrum. Methods Phys. Res., Sec. A 462, 337 (2001).

${ }^{26}$ F. Furuta, T. Nakanishi, S. Okumi, T. Gotou, M. Yamamoto, M. Miyamoto, M. Kuwahara, N. Yamamoto, K. Naniwa, K. Yasui et al., Nucl. Instrum. Methods Phys. Res., Sect. A 538, 33 (2005).

${ }^{27}$ A. Dangwal Pandey, G. Müller, D. Reschke, and X. Singer, Phys. Rev. Spec. Top. Accel. Beams 12, 023501 (2009). 
${ }^{28}$ M. BastaniNejad, M. A. Mohamed, A. A. Elmustafa, P. Adderley, J. Clark, S. Covert, J. Hansknecht, C. Hernandez-Garcia, M. Poelker, R. Mammei et al., Phys. Rev. Spec. Top. Accel. Beams 15, 083502 (2012).

${ }^{29}$ R. G. Forbes, C. Edgcombe, and U. Valdrè, Ultramicroscopy 95, 57 (2003).

${ }^{30}$ J. E. Inglesfield, Philos. Trans. R. Soc. London, Ser. A 334, 527 (1991).

${ }^{31}$ H. J. Kreuzer, L. C. Wang, and N. D. Lang, Phys. Rev. B 45, 12050 (1992).

${ }^{32}$ H. C. Miller, J. Appl. Phys. 38, 4501 (1967).

${ }^{33}$ G. Kresse and J. Hafner, Phys. Rev. B 47, 558 (1993).

${ }^{34}$ G. Kresse and J. Hafner, Phys. Rev. B 49, 14251 (1994).

${ }^{35}$ G. Kresse and J. Furthmüller, Comput. Mater. Sci. 6, 15 (1996).

${ }^{36}$ G. Kresse and J. Furthmüller, Phys. Rev. B 54, 11169 (1996).

${ }^{37}$ P. E. Blöchl, Phys. Rev. B 50, 17953 (1994).
${ }^{38}$ G. Kresse and D. Joubert, Phys. Rev. B 59, 1758 (1999).

${ }^{39}$ J. P. Perdew, K. Burke, and M. Ernzerhof, Phys. Rev. Lett. 77, 3865 (1996).

${ }^{40}$ J. P. Perdew, K. Burke, and M. Ernzerhof, Phys. Rev. Lett. 78, 1396 (1997).

${ }^{41}$ Y. Zhang and W. Yang, Phys. Rev. Lett. 80, 890 (1998).

${ }^{42}$ M. Methfessel and A. Paxton, Phys. Rev. B 40, 3616 (1989).

${ }^{43}$ C. Kittel, Introduction to Solid State Physics, 7th ed. (Wiley, 1996).

${ }^{44}$ N. D. Lang and W. Kohn, Phys. Rev. B 1, 4555 (1970).

${ }^{45}$ N. D. Lang and W. Kohn, Phys. Rev. B 7, 3541 (1973).

${ }^{46}$ R. G. Forbes, Ultramicroscopy 73, 31 (1998).

${ }^{47}$ R. G. Forbes, Ultramicroscopy 79, 25 (1999).

${ }^{48}$ E. Durand, Electrostatique et magnétostatique (Masson, 1953).

${ }^{49}$ T. Utsumi, IEEE Trans. Electron Devices 38, 2276 (1991). 